\title{
CORRIGENDUM
}

\section{Osteopontin signaling upregulates cyclooxygenase-2 expression in tumor-associated macrophages leading to enhanced angiogenesis and melanoma growth via $\alpha 9 \beta 1$ integrin}

S Kale, R Raja, D Thorat, G Soundararajan, TV Patil and GC Kundu

Oncogene (2015) 34, 5408-5410; doi:10.1038/onc.2015.315

Correction to: Oncogene (2014) 33, 2295-2306; doi:10.1038/ onc.2013.184; published online 3 June 2013

Since the publication of the above article the authors have identified an inadvertent mistake in Figures $2 \mathrm{c}$ and $4 \mathrm{~d}$. Due to unintentional errors made during the preparation of figures, duplication of certain images (control data) has occurred in these figures.

The authors wish to apologise for any inconvenience caused. 
Figure $2 c$ depicts a macrophage migration in response to melanoma cell secretary factors. While assembling the images, the authors inadvertently substituted the untreated (Un) and control siRNA (Coni) images with COX2 CDNA and OPN CDNA images.

Below is the corrected version of Figure 2c with images of Un and Coni. For quantification in this experiment, appropriate images were used and thus there is no need to change the graph.
In Figure 4d, the authors made errors in choosing some of the representative images from the appropriate experimental group in the tube formation experiment. The corrected version of Figure $4 d$ is provided below with proper images as well as the graph that is presented as Figure 4e.

Despite the errors that occurred, the key findings and conclusions of this paper remain unchanged. a

RAW264.7

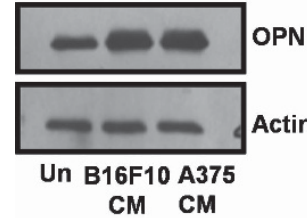

RAW264.7

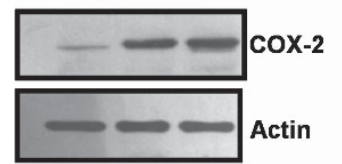

Un B16F10 A375

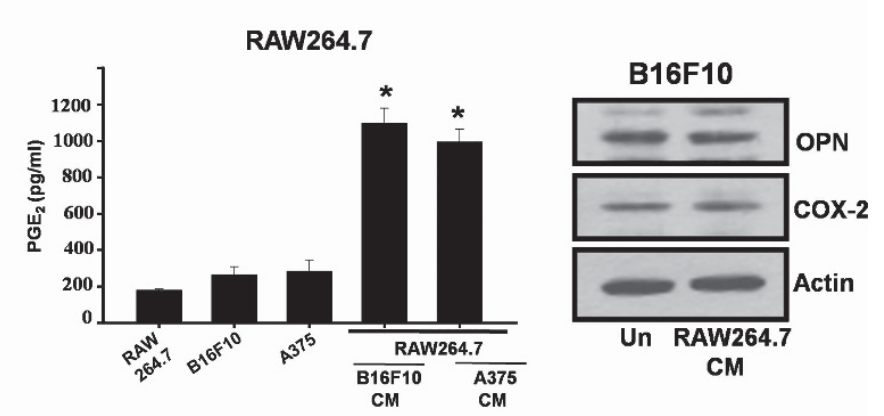

b

RAW264.7 (Upper chamber)
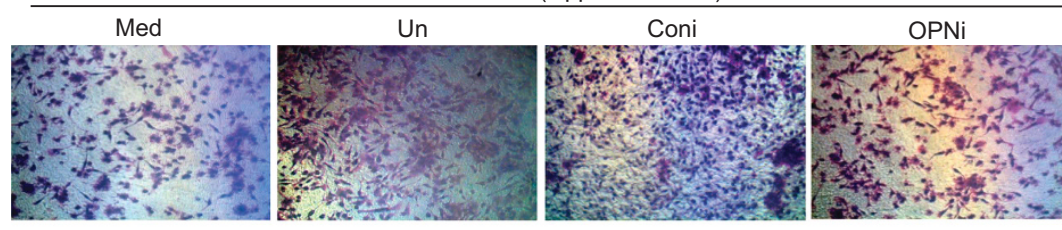

B16F10 CM (Lower chamber)

RAW264.7 (Upper chamber)

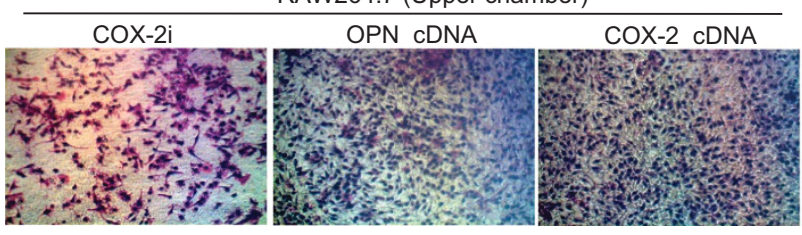

B16F10 CM (Lower chamber)

d
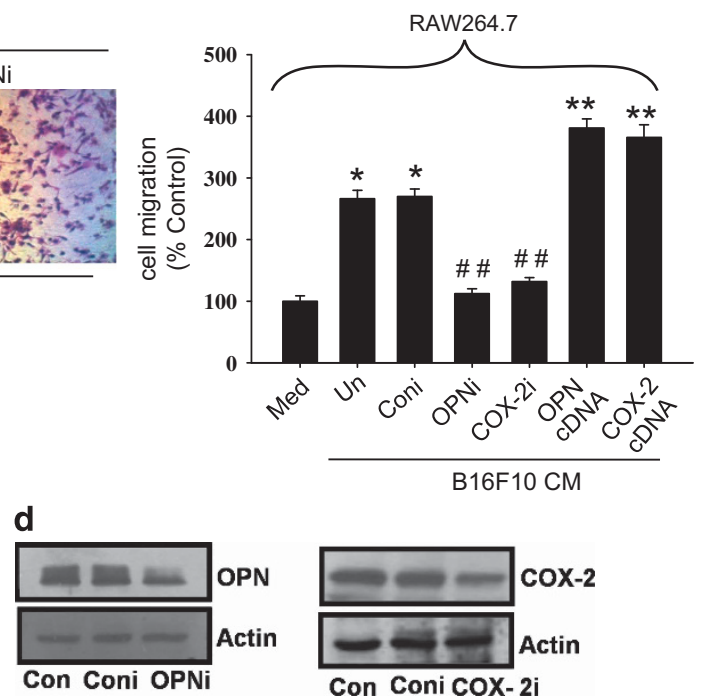

Figure 2. Co-culture and co-migration of macrophages (RAW264.7) with melanoma (B16F10 and A375) cells upregulate OPN and COX-2 expression. (a) RAW264.7 cells were supplemented with CM of melanoma. Expressions of OPN and COX-2 were analyzed by western blot. Actin was used as loading control (upper and lower panels). $P_{G E}$ was estimated by EIA and represented in the form of bar graph, ${ }^{*} P=0.008$ (right panel). (b) B16F10 cells were supplemented with CM of RAW264.7 and expressions of OPN and COX-2 were analyzed by western blot. (c) RAW264.7 $\left(1 \times 10^{6}\right)$ cells either alone or transfected with OPNi, COX-2i, OPN cDNA or COX-2 cDNA were used in the upper chamber, whereas CM of melanoma was added in the lower chamber. Migrated cells were stained, photographed (left panel) and migration of RAW264.7 cells in response to $\mathrm{CM}$ of B16F10 were quantified and represented in the form of bar graph. The error bar represents s.e.m., ${ }^{*} P<0.001$ versus Med; ${ }^{\# \#} P<0.001$ versus $C M$ of $B 16 \mathrm{~F} 10$. ${ }^{* *} P<0.005$ versus $C M$ of $B 16 \mathrm{~F} 10$ (right panel). All data are representation of three experiments. (d) Expressions of OPN and COX-2 in cells transfected with their specific siRNA were analyzed by western blot. Actin was used as control (left and right panels). 
a

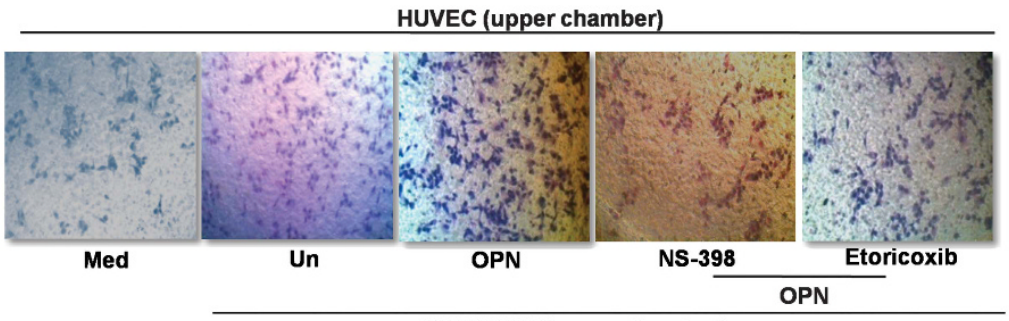

RAW264.7 (Lower chamber)

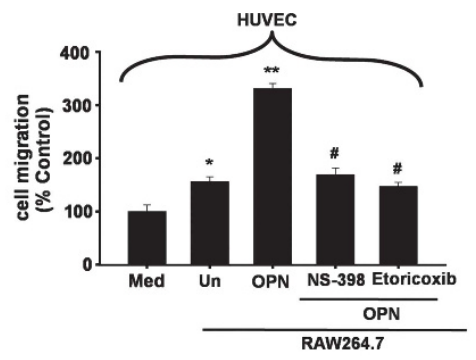

b

HUVEC

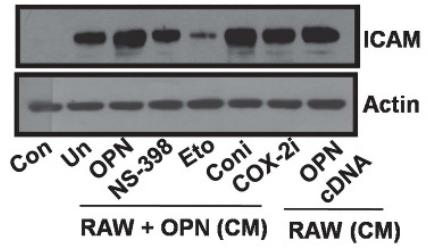

HUVEC

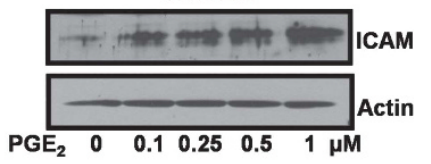

\section{C} HUVEC

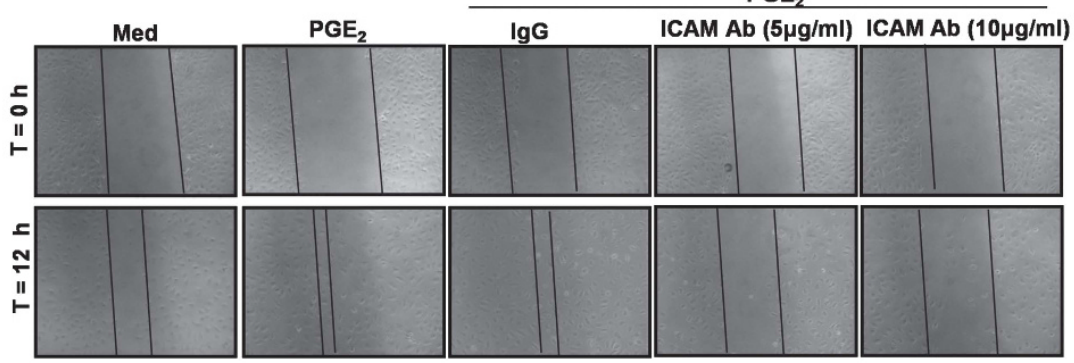

d

HUVEC

RAW264.7 CM
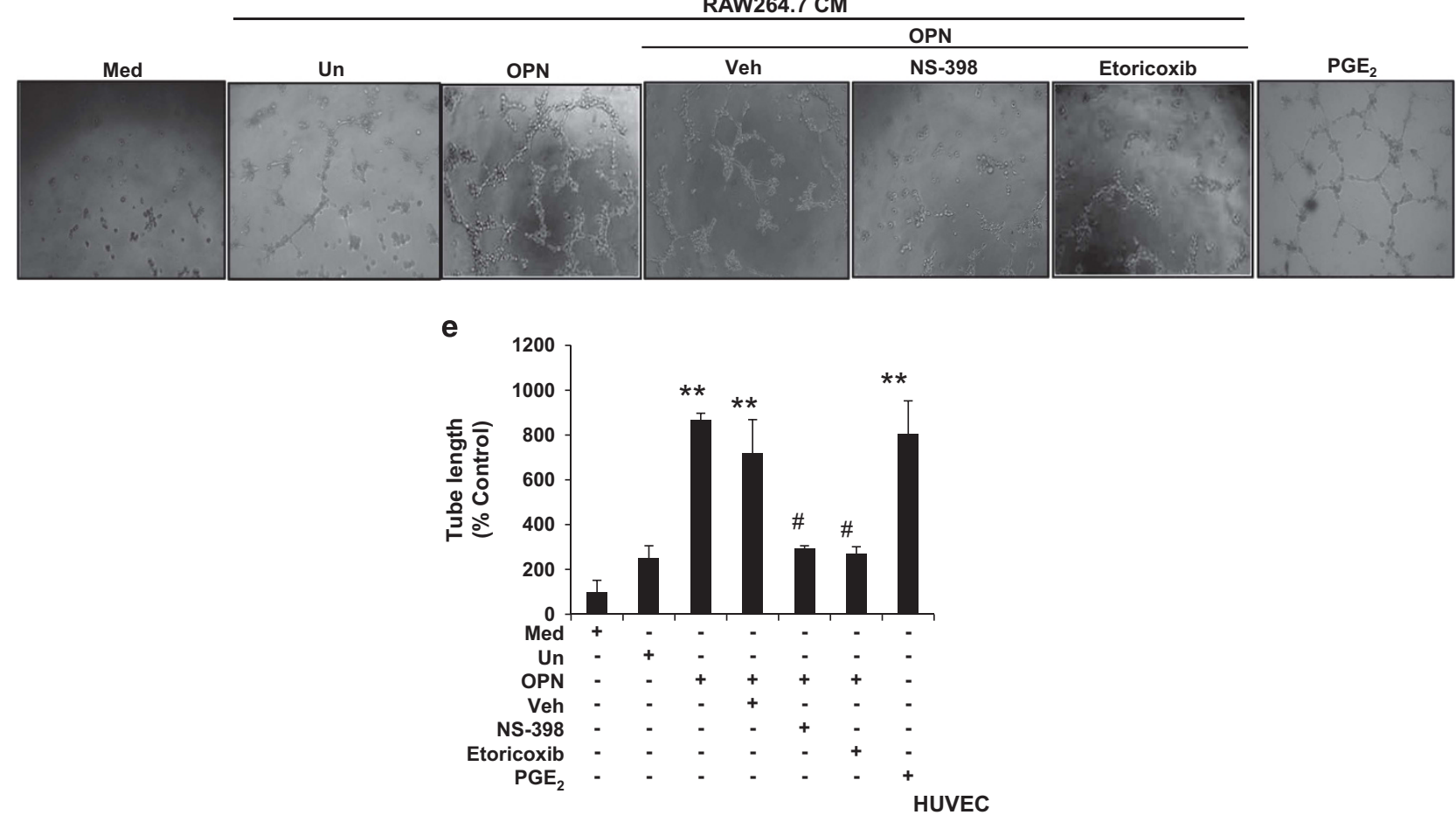

Figure 4. OPN-activated macrophages trigger ICAM-dependent HUVEC migration and promote tube formation via PGE 2 . (a) RAW264.7 cells, either treated with OPN alone or pretreated with NS-398 $(10 \mu \mathrm{M})$, Etoricoxib $(10 \mu \mathrm{m})$ and then treated with OPN $(0.5 \mu \mathrm{m})$, were used in the lower chamber, whereas HUVEC were used in the upper chamber. HUVEC migrated toward reverse side of the upper chamber were stained and photographed (left panel). Migration of HUVEC in response to CM of OPN-activated macrophages were quantified and represented in form of bar graph; Error bars, \pm s.e.m., ${ }^{*} P=0.025$ versus Med; ${ }^{* *} P<0.001$ versus untreated macrophages; $P<0.001$ versus CM of OPN-activated macrophages (right panel). (b) HUVEC were supplemented with CM of RAW264.7 cells treated with OPN either alone or along with NS-398, Etoricoxib or transfected with COX-2 siRNA or OPN CDNA. ICAM expression was analyzed by western blot (upper panel). HUVEC were treated with PGE 2 and expression of ICAM was analyzed by western blot (lower panel). (c) Wounds were made and HUVEC were pretreated with two doses of ICAM blocking antibody and then treated with $\mathrm{PGE}_{2}$. Wound photographs were taken after $12 \mathrm{~h}$. (d) HUVEC $\left(1 \times 10^{4}\right)$ were seeded on matrigel-coated plate and then supplemented with CM of RAW264.7 cells either treated with OPN alone or pretreated with NS-398, Etoricoxib and then treated with OPN $(0.5 \mu \mathrm{m})$. PGE 2 was used as positive control. After $4 \mathrm{~h}$ of incubation, endothelial cell tubular structure formation was photographed and analyzed. (e) Tube formation assay from panel $\mathbf{d}$ was quantified using Image Pro Plus 6.0 Software and represented graphically. Error bar represents s.e.m., ${ }^{* *} P \leqslant 0.01$ versus untreated macrophages $(U n) ;{ }^{\#} P<0.0001$ versus CM of OPN activated macrophages. 